\title{
BMJ Open Methodological issues in the design and evaluation of supported communication for aphasia training: a cluster-controlled feasibility study
}

\author{
Simon Horton, ${ }^{1}$ Allan Clark, ${ }^{2}$ Garry Barton, ${ }^{2}$ Kathleen Lane, ${ }^{1}$ Valerie M Pomeroy ${ }^{1}$
}

To cite: Horton S, Clark A, Barton $\mathrm{G}$, et al.

Methodological issues in the design and evaluation of supported communication for aphasia training: a clustercontrolled feasibility study. BMJ Open 2016;6:e011207. doi:10.1136/bmjopen-2016011207

- Prepublication history and additional material is available. To view please visit the journal (http://dx.doi.org/ 10.1136/bmjopen-2016011207).

Received 20 January 2016 Revised 22 March 2016 Accepted 24 March 2016

CrossMark

\footnotetext{
${ }^{1}$ Acquired Brain Injury Rehabilitation Alliance (ABIRA), School of Health Sciences, Queen's Building, University of East Anglia, Norwich, UK

${ }^{2}$ Norwich Medical School, University of East Anglia, Norwich, UK
}

Correspondence to Dr Simon Horton; s.horton@uea.ac.uk

\section{ABSTRACT}

Objective: To assess the feasibility and acceptability of training stroke service staff to provide supported communication for people with moderate-severe aphasia in the acute phase; assess the suitability of outcome measures; collect data to inform sample size and Health Economic evaluation in a definitive trial.

Design: Phase II cluster-controlled, observer-blinded feasibility study.

Settings: In-patient stroke rehabilitation units in the UK matched for bed numbers and staffing were assigned to control and intervention conditions.

Participants: 70 stroke rehabilitation staff from all professional groups, excluding doctors, were recruited. 20 patients with moderate-severe aphasia were recruited. Intervention: Supported communication for aphasia training, adapted to the stroke unit context versus usual care. Training was supplemented by a staff learning log, refresher sessions and provision of communication resources.

Main outcome measures: Feasibility of recruitment and acceptability of the intervention and of measures required to assess outcomes and Health Economic evaluation in a definitive trial. Staff outcomes: Measure of Support in Conversation; patient outcomes: Stroke and Aphasia Quality of Life Scale; Communicative Access Measure for Stroke; Therapy Outcome Measures for aphasia; EQ-5D-3L was used to assess health outcomes.

Results: Feasibility of staff recruitment was demonstrated. Training in the intervention was carried out with 28 staff and was found to be acceptable in qualitative reports. 20 patients consented to take part, 6 withdrew. 18 underwent all measures at baseline; 16 at discharge; and 14 at 6-month follow-up. Of 175 patients screened $71 \%$ were deemed to be ineligible, either lacking capacity or too unwell to participate. Poor completion rates impacted on assessment of patient outcomes. We were able to collect sufficient data at baseline, discharge and follow-up for economic evaluation.

Conclusions: The feasibility study informed components of the intervention and implementation in day-to-day practice. Modifications to the design are needed before a definitive cluster-randomised trial can be undertaken.

Trial registration number: ISRCTN37002304; Results.

\section{Strengths and limitations of this study}

- We report the findings of a feasibility study of a supported communication for aphasia intervention in an in-patient stroke rehabilitation setting. Despite the documented need for expert communication skills in working with people with aphasia this is the first study to investigate the methodological issues in the design and evaluation of supported communication for aphasia training in this context.

- Owing to the exploratory nature of the study we were able to make amendments to the protocol in response to new information as the trial progressed.

- Valuable insights gained into components of the intervention and its implementation will enable us to make necessary adjustments to the study design if a future trial is undertaken.

- We were unable to make power calculations for the chosen outcome measures.

- We did not use randomisation, so cannot judge acceptability of this to centres in a future trial.

\section{INTRODUCTION}

About 152000 people have a stroke in the UK each year. ${ }^{1}$ Approximately one-third experience aphasia, ${ }^{2}$ which can impair speaking, listening, reading and/or writing. Rehabilitation, care and safety of people with aphasia may be adversely affected by communication 'barriers' arising from the physical environment, negative staff attitudes or unskilled communication partners. ${ }^{3-5}$ Effective communication is essential to maintaining patient safety, ${ }^{6}$ ensuring a good experience of care, ${ }^{7}$ and providing the basis for therapeutic engagement and participation in decision-making through partnerships with healthcare professionals. ${ }^{8} 9$

Participation in conversational interactions is demonstrably enhanced for people with aphasia by providing 'supported communication' (SC). ${ }^{10}{ }^{11} \mathrm{SC}$ is premised on the view 
that interactional communication is collaborative, ${ }^{12}$ with the unimpaired communication partner (eg, healthcare practitioner) jointly responsible for achieving exchange of information and sustaining participation through adaptations to usual communication practices, for example the use of appropriately phrased questions, extra time or low-tech resources (eg, pen and paper)..$^{1013}$

SC for aphasia training is a complex intervention, ${ }^{14}$ including: interacting components (eg, staff training and subsequent skills-transfer to day-to-day practice); a range of behaviours required by those delivering (eg, staff communication practices) and receiving (eg, patient responses and behaviours) the intervention; a number of outcomes (eg, staff skills; patient participation, psychosocial or quality of life impact); and a degree of flexibility (eg, adapting the intervention according to clinical context). ${ }^{14}$

A systematic review of communication partner training for aphasia concluded that partners can be trained to facilitate and support the communication of people with aphasia. $^{11}$ However, very few studies of healthprofessional communication partner training exist; there is a lack of investigation in the first 4 months after stroke; and a lack of information on the impact of partner training on psychosocial and quality of life outcomes for people with aphasia. There are no studies examining cost or economic impact. ${ }^{11}$ More recently, single-group pre-post studies of nurse and nursing assistant training have shown positive impacts on staff knowledge and awareness of aphasia, ${ }^{15}{ }^{16}$ staff-reported levels of patient frustration, ${ }^{15}$ staff-patient relationships ${ }^{16}$ and patients' self-reported communication. ${ }^{16}$

Changing behaviour in healthcare settings is particularly challenging due to the complex relationships between organisations, professionals and patients. ${ }^{17}$ Communication skills training alone may not lead to improved patient outcomes without attention to the needs of the practice setting and strategies for sustained implementation of the intervention in context. ${ }^{418}$

While SC for aphasia training has the potential to improve the participation, well-being and quality of life of people with aphasia, ${ }^{19}$ key uncertainties in developing and evaluating this intervention must be addressed before a definitive RCT is undertaken. These include: staff recruitment and training under control and experimental conditions; transfer of training to day-to-day practice; patient recruitment and retention early after stroke; adherence to and acceptability of the intervention; selection of suitable staff and patient outcome measures; co-ordination of all intervention components within a multicentre trial.

Guided by recommendations for developing and evaluating complex interventions ${ }^{14}$ and reporting feasibility work ${ }^{20}$ we examined these methodological issues in order to assess the feasibility of conducting a subsequent trial of supported communication training for patients early after stroke in mutlidisciplinary stroke teams.
METHODS

\section{Design}

A cluster-controlled observer-blinded design was used in line with Medical Research Council (MRC) recommendations, ${ }^{21}$ where the intervention targets health professionals in order to study the subsequent impact on patient outcomes, protecting against contamination across trial groups where patients are managed within the same setting. ${ }^{22}$ Qualitative interviews, focus groups and ethnographic methods were used to investigate staff experiences and transfer of training to the workplace.

\section{Settings}

Two in-patient stroke rehabilitation units were identified, matched on the basis of: bed numbers; staffing levels; and estimated number of people with stroke admitted each year. We further determined similarity between units by collecting anonymised routine demographic and clinical discharge data on consecutive patients over a 5-month period before the trial onset (34 control and 28 intervention patients). There were no significant differences in: patient demographic characteristics; Therapy Outcome Measures (aphasia), ${ }^{23}$ impairment and activity scores at admission and discharge; mean length of stay (LOS) on the units; distribution of stroke types (lacunar stroke (LACS); partial anterior infarct (PACS); posterior circulation stroke (POCS); and total anterior circulation infarct (TACS)). Only 'LOS on previous (acute) unit' was significantly higher for the intervention site $(11(6-18)$ vs $5.5(2.5-9)$ days, $\mathrm{p}=0.0002)$. Units were assigned to control and intervention site, based on the stroke unit from which staff and patient participants were recruited.

\section{Patient and public involvement}

Patient and public involvement (PPI) was integral to the study from bid development through trial conduct and dissemination. A collaborating group of people with aphasia prioritised staff training as a focus for the bid; conducted staff skills assessment, were involved in refining and delivering the intervention, and in disseminating findings. Two PPI collaborators were members of the Trial Steering Committee.

\section{Ethics and informed consent}

Ethical approval for the study was granted by the local research ethics committee (ref: 10/H0310/69). All ethical and research governance procedures were in place before the study began. All staff and patient participants gave individual written consent to take part in the study.

\section{Participants and sample size}

As this was a feasibility study, a power calculation was not conducted. A two-site study was considered appropriate to address the feasibility aims. 


\section{Staff}

We aimed to recruit 24 staff from each setting, approximately $50 \%$ of all staff per unit, drawn proportionately from: nurses (day shifts only, Bands 5-7); qualified therapy staff (Bands 5-7)—occupational therapists (OTs); physiotherapists (PTs); speech and language therapists (SLTs); therapy/healthcare assistants (Bands 2-4). Medical staff were excluded because rotation of junior doctors made it unlikely they could complete the study.

\section{Patients}

We aimed to recruit 50 patients from each unit. This number exceeds the minimum specified in guidance, ${ }^{24}$ however it was felt appropriate to recruit more due to: (1) the clustered nature of this study; (2) non-random allocation of individuals; (3) anticipated high drop-out rate; (4) heterogeneity of stroke survivors.

\section{Inclusion criteria}

People aged 18+ years, who have had a stroke (first or recurrent) and have moderate-severe aphasia established by a SLT using the Therapy Outcome Measures (TOMS (aphasia) (impairment and activity score 0-3.5). ${ }^{23}$ We aimed to recruit patients with moderate-severe aphasia, because SC has been shown to be most effective with this group, compared with patients with mild aphasia, who are not thought to benefit. ${ }^{25}$

Aphasia type: expressive; receptive; or both, all at moderate or severe level as assessed on TOMS (aphasia).

Able to give informed written or witnessed oral consent as judged by an SLT or other qualified member of the clinical team (eg, OT; clinical psychologist).

\section{Interventions}

During standard clinical practice communication skills and strategies are used in order to enhance provision of all aspects of rehabilitation and care. This standard communication activity, therefore, continued for each patient, irrespective of intervention allocation, from admission until discharge, according to their needs at any one time. Staff delivering the intervention or standard clinical practice were separate from research staff, who provided training and recorded outcome measurements.

\section{Control intervention}

Usual communication practice during routine rehabilitation and care: stroke staff education for patients' communication needs as recommended in clinical guidelines. ${ }^{9}{ }^{26}$ Training in communication with people with aphasia on the control unit was individualised to specific staff and patient needs at the clinical team's discretion, and carried out 'hands-on' in routine clinical practice settings. All routine care and rehabilitation activities continued.

\section{Experimental intervention}

The experimental intervention, SC skills training, was adapted to a multidisciplinary stroke rehabilitation context from existing approaches ${ }^{13} 27$ through collaborative involvement of staff and service users (PPI). This resulted in a training resource based on the principles, values and practices of SC ('Supporting communication for access and participation'-details of content and procedures used in individualised staff training in online supplementary file 1), with a particular focus on knowing the person and building a supportive relationship. ${ }^{8}$ Training lasting approximately $4 \mathrm{~h}$ was delivered in small multidisciplinary staff groups ; $3 \mathrm{~h}$ devoted to theoretical aspects, followed for each staff member by two half-hour 1:1 experiential training sessions with a Conversation Partner Trainer, a person with aphasia 'trained to train' and give feedback to staff on their skills and use of resources.

\section{Outcome assessment}

Outcome and process measures and procedures were chosen to assess the feasibility of the study design. We collected recruitment data for staff and patients on each unit. To measure the impact of training on staff SC skills we used the measure of support in conversation (MSC), ${ }^{25}$ applied to $10 \mathrm{~min}$ video recorded interactions of individual staff with volunteers with aphasia. To assess change that might result from enhanced participation, and which patients might consider important in terms of quality of life and well-being, ${ }^{19}$ we used the Stroke and Aphasia Quality of Life Scale (SAQOL-39g). ${ }^{28}$ To measure patient satisfaction with staff communication in ways that might reflect the quality of support for patient engagement and participation we used the Communicative Access Measure for Stroke (CAMS3): patient satisfaction instrument developed by the Aphasia Institute in Toronto (http://www. aphasia.ca/). To measure impairment, activity, participation and well-being for anonymised cases we used the TOMS (aphasia) Impairment, Activity, Participation and Well-Being scores ${ }^{23}$ collected at admission and discharge. The EQ-5D-3L, ${ }^{29}$ was used to assess health outcomes.

Mixed qualitative methods were used to examine transfer of training to everyday practice in the intervention site: contemporaneous experiences of implementing SC were collected using a monthly 'learning log'; staff (Bands 2-7) perspectives on training and implementation were collected in two focus groups $(\mathrm{N}=5$ staff in total) and interviews $(\mathrm{N}=6)$ at the end of the study; data on patient participation, engagement and communication support were collected through maximum variation sampling of video recorded observations of day-to-day practice with six patients in-interaction with Nurse, PT, OT, SLT, health care assistant (HCA), nonclinical assistant (NCA) staff (Bands 2-6) on 12 occasions.

\section{Analysis}

To assess reliability of MSC rating between two raters in the first instance, a two-way random effects analysis of variance was used and the ICC was reported. All analyses were carried out in Stata/SE V.11.2. 
Staff and patient characteristics in the two study arms were compared using a $\chi^{2}$ test for binary or categorical outcomes and a t test or Mann-Whitney test if the outcome measure was continuous. Owing to each intervention having only one cluster it was not possible to account for clustering in the analysis. Although $p$ values were calculated for comparisons the main purpose of the analysis is to describe the two arms rather than draw inferences from the data.

The patient outcomes SAQOL-39g and EQ-5DL were compared between the control and intervention groups at baseline, discharge and 6-month follow-up; CAMS3 was compared only at discharge. For anonymised cases TOMS Impairment, Activity, Participation and Well-Being scores were compared at admission and discharge.

Qualitative thematic analysis was used for learning-log text, focus group and interview data, ${ }^{30}$ video data were examined using activity analysis, ${ }^{31}$ and conversation analysis. ${ }^{32}$

Economic analysis focused on completion rates and cost drivers. Costs (based on self-reported levels of resource use at 6-month follow-up) were calculated from the perspective of the National Health Service (NHS) and personal social services (PSS) (estimated at 2011/2012 financialyear levels), encompassing those costs that were related to the intervention. A preliminary within-trial analysis (based on a complete case analysis) was also conducted over a 6-month period, where Quality Adjusted Life Year (QALY) scores were calculated for those who completed the EQ-5D-3L at baseline and 6-month follow-up and the incremental cost/effect was based on the mean (unadjusted) difference between groups.

\section{RESULTS}

A number of amendments to the trial design and procedures were introduced over the course of the trial (see online supplementary file 2); for the final protocol version see online supplementary file 3 .

\section{Feasibility}

Staff recruitment and retention

Staff were recruited to target (24 per unit) within 4 (control) and 3.5 (intervention) months; additional staff were subsequently recruited to cover attrition. Recruitment rates were slower than anticipated: institutional barriers (eg, staff shift patterns) and inconsistent lines of communication prevented timely access to staff. In total we recruited 33 control and 37 intervention unit staff. Five control staff withdrew and three were unable to complete measures; five intervention staff withdrew and five were unable to complete measures. Overall, recruitment was from nursing (27\%); therapy $(17 \%)$; HCAs (31\%); associate practitioners (APR) (28\%); others $(10 \%)$. Fifty-six per cent came from Bands 2-4; $44 \%$ from Bands 5-7; 26\% of staff had English as a second language. Staff characteristics between units did not differ significantly, apart from more part-time staff at the intervention site $(\mathrm{p}=0.011)$; see table 1 .
Patient recruitment and retention

In total 175 patients with aphasia were screened over a 20-month period (see figure 1); $47(28.86 \%)$ were eligible for the study; $20(42.5 \%)$ consented to participate (13 intervention; 7 control); due to slow recruitment, the recruitment period was extended from 14 to 20 months. Of the 128 non-eligible patients, 25 (19.5\%) did not meet the inclusion criteria; 59 (46\%) were deemed unable to give consent; and $44(34 \%)$ were ineligible due to other causes (eg, failure to recruit).

Six patients withdrew due to distress or fatigue during the in-patient episode, or ill-health at follow-up. No significant differences presented between units in terms of gender, age, admission TOMS or LOS of recruited patients. Baseline characteristics are given in table 2.

\section{Anonymised patient cases}

We collected anonymised demographic and clinical data for in-patient episodes on 55 (control) and 44 (intervention) patients over a 9-month period. The units were equivalent in terms of patient demography, stroke type

\begin{tabular}{|c|c|c|c|}
\hline & $\begin{array}{l}\text { Control } \\
(n=33)\end{array}$ & $\begin{array}{l}\text { Intervention } \\
(\mathrm{n}=37)\end{array}$ & p Value \\
\hline Male & $12(36.4 \%)$ & $8(21.6 \%)$ & $0.173^{\star}$ \\
\hline Professional group & & & $0.405 \uparrow$ \\
\hline Nurse & $6(18.2 \%)$ & $13(35.1 \%)$ & \\
\hline OT & $2(6.1 \%)$ & $3(8.1 \%)$ & \\
\hline PT & $2(6.1 \%)$ & $2(5.4 \%)$ & \\
\hline SLT & $1(3.0 \%)$ & 2 (5.4\%) & \\
\hline $\mathrm{HCA}$ & $11(33.3 \%)$ & $11(29.7 \%)$ & \\
\hline APR & 15 (15.2\%) & 5 (13.5\%) & \\
\hline NT & 4 (12.1\%) & 0 & \\
\hline Other & $2(6.1 \%)$ & $1(2.7 \%)$ & \\
\hline Band & & & $0.206 \ddagger$ \\
\hline 2 & $11(33.3 \%)$ & $10(27.0 \%)$ & \\
\hline 3 & $6(18.2 \%)$ & $3(8.1 \%)$ & \\
\hline 4 & 5 (15.2\%) & $4(10.8 \%)$ & \\
\hline 5 & $6(18.2 \%)$ & $13(35.1 \%)$ & \\
\hline 6 & $4(12.1 \%)$ & $5(13.5 \%)$ & \\
\hline 7 & $1(3.0 \%)$ & $2(5.4 \%)$ & \\
\hline Part-time & $2(6.1 \%)$ & $11(29.7 \%)$ & $0.011^{*}$ \\
\hline Bank staff & $1(3.0 \%)$ & $5(13.5 \%)$ & 0.118 \\
\hline $\begin{array}{l}\text { English as first } \\
\text { language }\end{array}$ & $25(75.8)$ & $27(77.1 \%)$ & 0.893 \\
\hline $\begin{array}{l}\text { Months of stroke } \\
\text { experience } \\
\text { (median, IQR) }\end{array}$ & $24(2-60)$ & $20(18-36)$ & 0.4740 \\
\hline \multicolumn{4}{|c|}{$\begin{array}{l}\text { Bands } 2-7 \text { indicate (from low=2 to high=7) seniority and pay scale } \\
\text { grades. } \\
\text { Bank staff are those not employed by the unit, but who work } \\
\text { occasional hours. } \\
\text { ` } \chi^{2} \text { test. } \\
\text { tFisher's exact test. } \\
\text { fMann-Whitney test. } \\
\text { APR, associate practitioner; HCA, health care assistant; NT, } \\
\text { nutritional assistant; OT, occupational therapist; PT, } \\
\text { physiotherapist; SLT, speech and language therapist. Others } \\
\text { included non-clinical assistant, OT and PT assistant. }\end{array}$} \\
\hline
\end{tabular}


Figure 1 CONSORT flow

diagram of the trial conduct. CAMS3, Communicative Access

Measure for Stroke; HSU Q;

Health Services Use

Questionnaire, SAQOL, Stroke and Aphasia Quality of Life Scale.

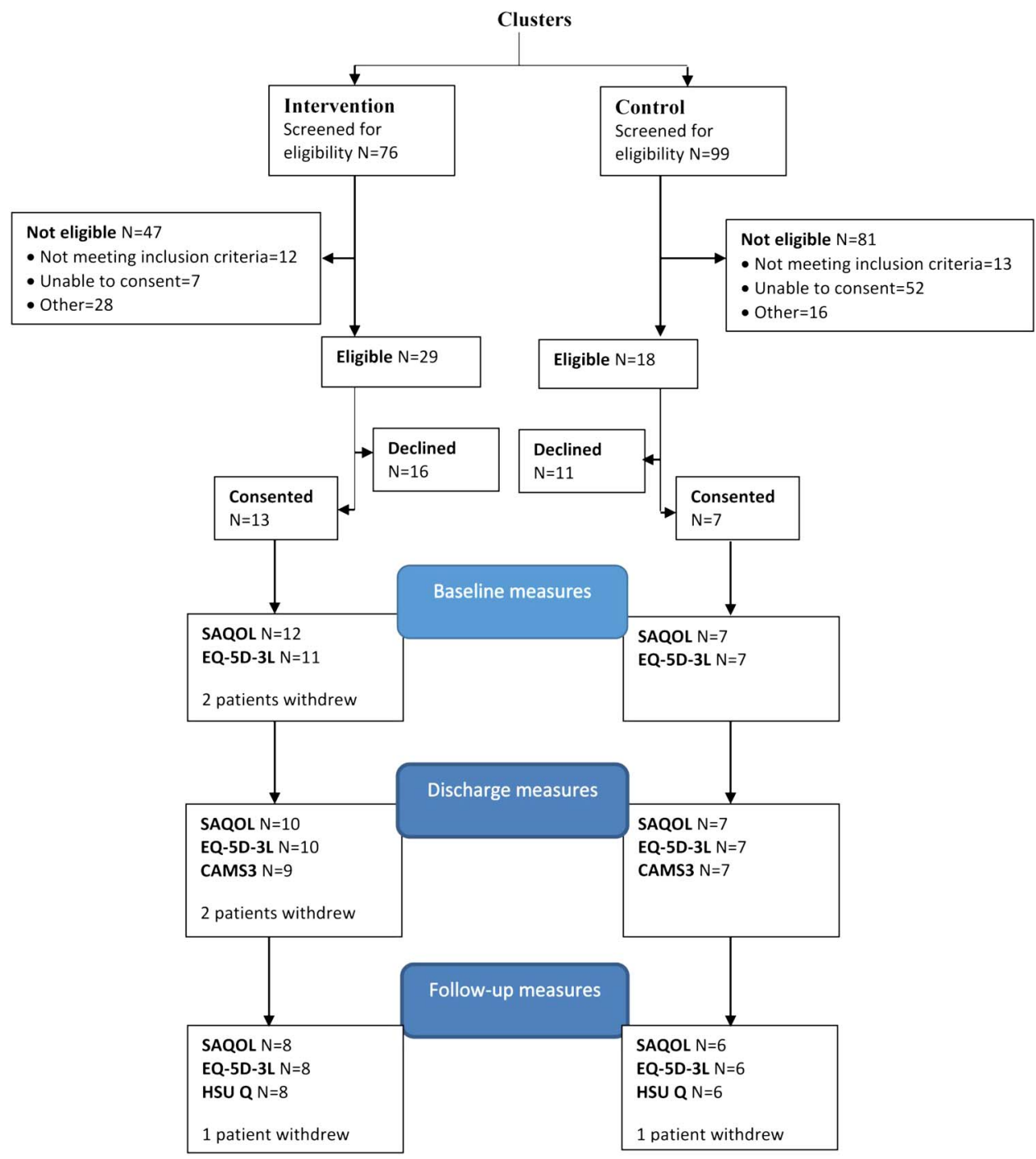

and LOS; however, intervention-unit patients had higher scores at admission on all components of the TOMS (table 3).

\section{Feasibility of staff training}

Training was provided for 3 control and 28 intervention staff (9 nursing; 2 PT; 2 OT; 1 SLT; 9 HCA; 4 APR;

Table 2 Summary of recruited patients' baseline characteristics by site

\begin{tabular}{|c|c|c|c|}
\hline & $\begin{array}{l}\text { Control } \\
(n=7)\end{array}$ & $\begin{array}{l}\text { Intervention } \\
(n=13)\end{array}$ & p Value \\
\hline Male & $5(71.4 \%)^{\star}$ & $6(46.2 \%)$ & 0.279 \\
\hline Age (years) & $62(22.0)$ & $71.6(15.3)$ & 0.265 \\
\hline \multicolumn{4}{|c|}{ TOMS† (Adm.) } \\
\hline I & $3(2.5-3)$ & $3(1-3)$ & 0.690 \\
\hline A & $2.5(2-3)$ & $3(1.5-3.5)$ & 0.249 \\
\hline LOS on unit & $43.6(15.6)$ & $49.0(17.2)$ & 0.509 \\
\hline \multicolumn{4}{|c|}{$\begin{array}{l}\text { *Values are } n(\%) \text { for binary/categorical variables and either mean } \\
\text { (SD) or median (IQR) for continuous outcomes. } \\
\text { tTherapy Outcome Measures at Admission. } \\
\text { A, activity; I, impairment; LOS, length of stay. }\end{array}$} \\
\hline
\end{tabular}

1 NCA). Resource limitations in clinical teams restricted provision of control-unit training. Shift patterns, staff shortages or lack of room availability prevented timely provision of training for intervention unit staff, which took place over eight compared with the three planned sessions.

\section{Transfer of training to day-to-day practice}

During the course of the trial we developed methods for ongoing staff support for implementation: (1) a monthly reflective learning log (see online supplementary file 4); (2) 'nudges' in the form of a pocket guide (see online supplementary file 5), 'supporting communication' pens as a reminder to use the intervention, and a ward-based folder summarising key points of SC. We offered two 'refresher sessions' for staff, but these were very poorly attended.

Transfer of training and implementation of SC in day-to-day practice is reported fully elsewhere. ${ }^{33}$ In summary: learning logs were completed by $46 \% \quad(\mathrm{~N}=17 /$ 37 ) of staff, with $31 \%(\mathrm{~N}=54 / 174)$ logs returned for analysis over a 10-month period. Analysis of learning log and focus group/interview data indicated that: (1) staff 
Table 3 Summary of anonymised routinely-collected data by site

\begin{tabular}{|c|c|c|c|}
\hline & Control $(n=55)$ & $\begin{array}{l}\text { Intervention } \\
(\mathrm{n}=44)\end{array}$ & p Value \\
\hline Male & $24(43.6 \%)^{*}$ & $23(52.3 \%)$ & $0.393 \dagger$ \\
\hline Age (years) & $76.6(14.4)$ & $75.4(12.3)$ & $0.661 \ddagger$ \\
\hline \multicolumn{3}{|l|}{ Stroke type } & $0.259 \S$ \\
\hline TACS & $27(49.1 \%)$ & $19(43.4 \%)$ & \\
\hline PACS & $24(43.6 \%)$ & $21(47.7 \%)$ & \\
\hline POCS & 0 & $1(2.3 \%)$ & \\
\hline LACS & $1(1.8 \%)$ & $3(6.8 \%)$ & \\
\hline Missing & $3(5.5 \%)$ & 0 & \\
\hline LOS on unit & $36.5(31.8)$ & $38.6(31.4)$ & $0.329 \rrbracket$ \\
\hline \multicolumn{4}{|c|}{ TOMS on admission } \\
\hline 1 & $1(0.5-3)$ & $3(1-3.5)$ & $0.003 \rrbracket$ \\
\hline$A$ & $1.5(0.5-3)$ & $3(1-4)$ & $<0.0019$ \\
\hline $\mathrm{P}$ & $1(0.5-3)$ & $3(1-4)$ & $0.003 \rrbracket$ \\
\hline WB & $3(2-4)$ & $4(3-4)$ & 0.018 1 \\
\hline \multicolumn{4}{|c|}{ TOMS on discharge } \\
\hline 1 & $2.75(1-3.5)$ & $3.25(1.5-4)$ & $0.061 \rrbracket$ \\
\hline$A$ & $2.5(1.25-3.5)$ & $3.5(2-4)$ & $0.012 \rrbracket$ \\
\hline $\mathrm{P}$ & $3(1.25-4)$ & $4(2-4)$ & $0.079 \rrbracket$ \\
\hline WB & $4(2-5)$ & $4(3-4.5)$ & $0.514 \rrbracket$ \\
\hline \multicolumn{4}{|c|}{ Change in TOMS } \\
\hline I & $0.5(0-1)$ & $0.25(0-0.5)$ & $0.119 \rrbracket$ \\
\hline$A$ & $0.5(0-1)$ & $0.25(0-1)$ & $0.214 \rrbracket$ \\
\hline $\mathrm{P}$ & $0.5(0-1.5)$ & $0(0-1)$ & $0.046 \rrbracket$ \\
\hline WB & $0(0-1)$ & $0(0-0.5)$ & $0.193 \rrbracket$ \\
\hline
\end{tabular}

${ }^{*}$ Values are $\mathrm{n}(\%)$ for binary/categorical variables and either mean (SD) or median (IQR) for continuous outcomes.

${ }^{*} \chi^{2}$ test.

tt test.

†Fishers exact test.

१Mann-Whitney test.

A, activity; I, impairment; LACS, lacunar stroke; PACS, partial anterior infarct; POCS, posterior circulation stroke; $\mathrm{P}$, participation; TACS, total anterior infarct; TOMS, Therapy Outcome Measures at admission; WB, Well-being.

applied skills learnt in training, but the need for more practice with trainers and feedback after video assessments was emphasised; (2) HCAs reported learning new information more frequently than therapy staff in Bands 5-7; (3) many staff felt more confident about working with people with aphasia; (4) more practice with and support for working with patients with severe aphasia and additional cognitive impairments was needed; (5) busy, noisy environments and time pressures increased problems for staff interacting with patients.

We were unable to collect video data of routine practice at the control site because no staff gave consent. Analysis of video data from the intervention site indicated appropriate use of supported communication, but across all Bands opportunities for full patient participation were not consistently realised; intrusive noise (eg, radio; TV; voices) was evident in most ward-based activities.

\section{Suitability of outcome measures}

Measure of support in conversation

Satisfactory inter-rater reliability on the MSC was not achieved, despite three attempts at assessor training and
Table 4 Summary of recruited individuals' SAQOL-39g data by site

\begin{tabular}{|c|c|c|c|}
\hline & Control & Intervention & p Value \\
\hline SAQOL (Adm.) & $\mathrm{n}=7$ & $n=12$ & \\
\hline Mean & $2.96(0.66)$ & $2.5(0.59)$ & 0.142 \\
\hline Physical & $3.2(0.78)$ & $2.39(0.93)$ & 0.070 \\
\hline Comm. & 2.03 (1.19) & $2.51(0.7)$ & 0.287 \\
\hline Psych. & $2.97(0.99)$ & $2.64(0.67)$ & 0.392 \\
\hline SAQOL (Discharge) & $\mathrm{n}=7$ & $n=10$ & \\
\hline Mean & $3.25(0.64)$ & $2.97(0.74)$ & 0.440 \\
\hline Physical & $3.79(0.58)$ & $2.48(0.7)$ & 0.001 \\
\hline Comm. & $2.72(1.13)$ & $3.24(1.16)$ & 0.370 \\
\hline Psych. & $2.86(0.84)$ & $3.35(0.87)$ & 0.264 \\
\hline SAQOL-F/U & $\mathrm{n}=6$ & $\mathrm{n}=8$ & \\
\hline Mean & $3.49(0.57)$ & $3.46(0.83)$ & 0.936 \\
\hline Physical & $3.74(0.83)$ & $3.25(1.26)$ & 0.422 \\
\hline Comm & $2.93(0.81)$ & $3.3(1.13)$ & 0.503 \\
\hline Psych & $3.53(0.47)$ & $3.77(0.94)$ & 0.582 \\
\hline \multicolumn{4}{|c|}{ SAQOL change to discharge } \\
\hline Mean & $0.29(0.55)$ & $0.45(0.74)$ & 0.634 \\
\hline Physical & $0.59(0.94)$ & $0.01(0.89)$ & 0.216 \\
\hline Comm. & $0.69(0.83)$ & $0.66(0.92)$ & 0.946 \\
\hline Psych. & $-0.12(0.42)$ & $0.77(0.98)$ & 0.040 \\
\hline \multicolumn{4}{|c|}{ SAQOL change to follow-up } \\
\hline Mean & $0.39(1.07)$ & $0.83(0.71)$ & 0.374 \\
\hline Physical & $0.5(1.45)$ & $0.6(0.79)$ & 0.877 \\
\hline Comm. & $0.73(1.03)$ & $0.62(0.8)$ & 0.827 \\
\hline Psych. & $0.26(0.98)$ & $1.16(1.19)$ & 0.155 \\
\hline
\end{tabular}

Values are $n(\%)$ for binary/categorical variables and either mean (SD) or median (IQR) for continuous outcomes.

Higher scores indicate more positive outcomes.

SAQOL-39g, Stroke and Aphasia Quality of Life Scale-39 item.

revisions to MSC descriptors. Individual video clips were rated by two raters on the MSC; best reliability levels achieved were: acknowledge competence ICC $=0.27$ (95\% CIs -0.12 to 0.64 ); reveal competence ICC $=0.29$ $(-0.12$ to 0.67$)$. Thus we were unable to carry out group comparisons. We therefore used alternative approaches to examining training effects: (1) a purposive sample of 24 pre-training and post-training video clips from the intervention group were subject to qualitative analysis; (2) clips were blindly assessed as being from before or after training by an experienced SLT who was not otherwise connected with the study; $83 \%$ of clips were correctly judged.

\section{SAQOL39g (quality of life)}

We collected data on all sections of the SAQOL39g from participants who remained in the study until follow-up and calculated change scores at discharge and follow-up. All recorded data collected for the SAQOL39g are set out in table 4 .

\section{CAMS3 (communicative access)}

CAMS3 was assessed at discharge only. We were able to collect complete data from all patients remaining in the study (table 5). 
TOMS Impairment Activity Participation and Well-Being (anonymised cases)

In collaboration with the clinical teams we collected routine TOMS data on admission and discharge for all aphasic patients at control and intervention sites (table 3).

Although some significant differences between control and intervention groups are evident, as well as within-group change scores on patient-related outcomes, due to the small sample size and the aims of the study, the summary statistics should be given more weight than the significance levels.

\section{Economic evaluation}

The training cost amounted to $£ 560.37$ per participant when apportioned across the 13 intervention participants who provided consent for the intervention. This was considered a conservative approach as we did not wish to underestimate such costs. Implementation (pocket guide and pens) costs were estimated at £33.23. For 22/48 responses practising supported communication did not change usual staff-patient consultation time; for the remainder there was a mean increase of $4.2 \mathrm{~min}$. Based on the assumption of two consultations per day for the duration of the original in-patient admission this amounted to a cost of $£ 233.33$ per participant. The total cost of the intervention was thereby estimated to be $£ 826.93$ per patient.

QALY scores were available for 13 patients (6 control, 7 intervention), and the mean scores was 0.018 higher in the intervention arm. For these intervention patients, the mean 6-month total NHS and PSS costs (including intervention costs) was £19688.86, compared to $£ 17999.72$ for the control patients, where admission costs are the largest cost-driver (the mean LOS was
$>40$ days). The preliminary within-trial incremental cost/ QALY estimate (which needs to be treated with caution due to the very small numbers) was $£ 96026$ ( $£ 1689.14$ / 0.018 ) and above the often-quoted $£ 20000$ figure. ${ }^{34}$ However, the potential for the intervention to be costeffective is demonstrated by a threshold analysis which shows that if the benefits are maintained and no further costs are incurred after the 6-month period, then the cost/QALY figure would fall below $£ 20000$ after a further 2.5 years.

\section{DISCUSSION}

We demonstrated the feasibility of recruiting staff to control and intervention conditions, and of delivering SC training to a multidisciplinary stroke team. We investigated patient eligibility and collected data on the feasibility of patient recruitment, outcome assessment and collection of data for HE evaluation. The study provided the opportunity to adapt the intervention to the context of in-patient stroke rehabilitation and involve service users in its design and delivery. We were able to gain valuable insights into how all components of the protocol worked together, including the logistics of conducting a multicentre trial.

\section{Strengths and limitations}

To the best of our knowledge this is the first study to test the feasibility of conducting a trial of SC training in an in-patient rehabilitation setting. Staff were recruited to target and retention was good, with only $14 \%$ withdrawing and $11 \%$ dropping out due to turnover. There was fair representation across professional groups, with over half from staff groups with high levels of patient contact

\begin{tabular}{lccc} 
Table 5 CAMS3 scores at discharge & & & \\
\hline & Control $(\mathbf{n}=\mathbf{7})$ & Intervention $(\mathbf{n}=\mathbf{9})$ & $\mathbf{p}$ Value \\
\hline Did the staff treat you with respect? & $1.5(1-2)$ & $2(2-2)$ & 0.110 \\
Did staff show that they understand your frustration? & $1.5(1.5-1.5)$ & $2(1.5-2)$ & 0.033 \\
Were staff sensitive? & $1.5(1-1.5)$ & $2(2-2)$ & 0.002 \\
Who helped & $1(1-1.5)$ & $1.5(1.5-2)$ & 0.088 \\
Doctors? & $1.5(1-2)$ & $1.5(1.5-2)$ & 0.363 \\
Nurses? & $1.5(1.5-2)$ & $2(1.5-2)$ & 0.155 \\
PT/OT? & $1.5(1.5-2)$ & $2(1.5-2)$ & 0.298 \\
SLT? & $1(0-1.5)$ & $1.5(1-2)$ & 0.130 \\
Understand info about & $1.5(0.5-2)$ & $1.5(1-2)$ & 0.545 \\
Health/medical? & $1(0-1.5)$ & $1(0.5-2)$ & 0.636 \\
Treatment? & $0.5(0-2)$ & $1(0-2)$ & 1.00 \\
Next steps/discharge? & $1(0.5-1.5)$ & $1.75(1.5-2)$ & 0.048 \\
Legal issues? & $1(0.5-1.5)$ & $1(1-2)$ & 0.672 \\
Did you get help to answer questions? & $0.5(0-1)$ & $1(0.5-2)$ & 0.109 \\
Did you get to give your own answers? & $0.5(0-1.5)$ & $1.25(0.75-1.5)$ & 0.366 \\
Could you ask questions about important things? & $1(0.5-2)$ & $2(0.5-2)$ & 0.696 \\
Could you join in discussions about you? & $1.5(1.5-2)$ & $1.5(1.5-2)$ & 0.820 \\
Overall, could you make your own decisions? & & \\
How satisfied were you with your experience here? & & & \\
\hline Vol & & & \\
\hline
\end{tabular}

Values are median and IQR; all $p$ values based on Mann-Whitney test Higher scores indicate more positive outcomes.

CAMS3, Communicative Access Measure for Stroke; OT, occupational therapists; PT, physiotherapists; SLT, speech and language therapists. 
(ie, Bands 2-4). Training in the intervention was generally viewed positively, with the involvement of trainers with aphasia highly regarded by staff; their inclusion throughout the study enabled us to ensure that training content was relevant to patient needs, and dissemination strategies reflected patient perspectives as well as academic and clinical ones (see online supplementary file 6). Transfer of training into day-to-day practice and use of the intervention were found to be acceptable; we developed good insights into how experiences varied across staff groups and grades, and were able to collect video-observations to assess adherence and explore aspects of practice not revealed through interviews or focus group discussions. Close collaboration with clinical teams enabled us to gain an understanding of patient eligibility and recruitment issues in the subacute stage; and to demonstrate the feasibility of collecting anonymised routine data relevant to rehabilitation outcomes, including psychosocial impact (TOMS Well-Being). The feasibility of conducting an economic evaluation was demonstrated by the reasonable response rates for completion of EQ-5D-3L at baseline, discharge and follow-up. The staff learning log was a novel approach not only to sustaining staff awareness of the intervention, but allowed us to collect data on the impact of the intervention on staff time and hence more accurate costs.

Although we were able to collect patient self-reported outcome data, there were insufficient numbers to conduct a power calculation. Many patients were deemed unable to consent or still too ill to be approached within the required time-frame, with $62 \%$ $(n=47 / 76)$ of those on the intervention unit and $82 \%$ $(n=81 / 99)$ on the control unit deemed ineligible. Pretrial anonymised clinical data showing higher 'LOS on previous (acute) unit' for intervention patients are open to a number of interpretations-for example, differences in local practices; location of acute and rehabilitation units; patients with greater recovery on admission to the rehabilitation unit. However, no other measures suggested systematic differences in patients admitted to the 2 units. On the other hand, anonymised data collected during the trial show significant differences in TOMS admission scores (table 3) between units, and a considerably higher proportion of patients were deemed to lack capacity on the control (64\%, $\mathrm{n}=52 / 81)$ compared with the intervention $(15 \%, \mathrm{n}=7 /$ 47) unit. We are unable to comment specifically on this finding as data were not prospectively and systematically collected on this aspect of the recruitment process. Attrition was another factor, with $6(30 \%)$ patient withdrawals, some due to fatigue and a lack of willingness to engage in lengthy self-report measures, even though we offered to complete these assessments over more than one session. Little training was provided in the control arm, and we were unable to make training effectiveness comparisons between groups due to problems establishing inter-rater reliability in the chosen outcome measure for this part of the study.

\section{Recruitment and training of staff}

Despite management buy-in, a commitment to the study at organisational level, ${ }^{35}$ and staff enthusiasm for being involved in research with the opportunity to gain new skills, ${ }^{33}$ there were considerable barriers to accessing staff in a timely fashion, due to shift patterns; lack of consistent avenues of communication; non-attendance; and lack of appropriate venues, all adding to overall training costs. Mandatory attendance or a whole-team training approach, ${ }^{4} 1633$ may have helped to reduce organisational barriers and costs, although natural staff turnover has the potential to affect the integrity of overall team skills levels. Limited training was provided for participating control staff, reflecting the pragmatic nature of this study. ${ }^{35}$

\section{Patient recruitment}

Recruitment and retention of patients in stroke trials is known to be challenging. ${ }^{36}$ Practical issues may delay the process of recruiting people with aphasia, ${ }^{37}$ despite their having capacity. ${ }^{38}$ In a recent study of early intervention in aphasia $92 \%$ of patients were recruited using next-of-kin consent. ${ }^{39}$ Despite a poor recruitment rate, we did recruit $43 \%(20 / 47)$ of eligible patients. This suggests that decision-making by gatekeepers, which has been found to affect recruitment in other stroke trials, ${ }^{40} 41$ or the nature of the organisations themselves, ${ }^{35}$ with earlier admission of more disabled patients, may have been key factors. Better preparation for gatekeepers and staff across participating units may have helped, including training in using TOMs, ${ }^{42}$ as a measure of comparability across patient cohorts. The focus on moderate-severe aphasia, which is linked to more severe strokes and greater levels of disability, may have impacted on recruitment and attrition, although Ali $e t a l,{ }^{38}$ found no such associations.

\section{Outcome measures}

The MSC may not be a suitable measure for use in assessing typical clinical interactions as it was developed in community settings with volunteers. ${ }^{10}{ }^{25}$ However, it has been used successfully in studies of supported communication training with volunteers, ${ }^{10}$ medical students ${ }^{43}$ and family members/partners. ${ }^{44}$ Eriksson et at $t^{45}$ tested the reliability and agreement of an adapted version of this scale and found that between 10 and $30 \mathrm{~h}$ of joint training were needed for assessors to reach satisfactory levels of reliability. In our study, we adapted some aspects of the MSC, but the two assessors received only $9 \mathrm{~h}$ of joint training over three sessions, which may have been insufficient.

The choice of self-report measures meant that patients must ideally consent to participation, implicitly impacting on our ability to collect sufficient data to carry out power calculations for SAQOL39g or CAMS3. Although use of such measures may place a high burden on patients' time or emotional resources during in-patient episodes, the importance of assessing the effects of 
supported communication interventions on well-being and quality of life has been emphasised. ${ }^{19}$ We demonstrated the feasibility of collecting routine anonymised patient data. Systematic collection of patient-relevant assessment data within stroke teams is recommended in clinical guidelines. ${ }^{26}$ Such data may be collected using observational approaches such as TOMS, which have the potential to be used in making unit-level comparisons. ${ }^{23}$ However, as generic outcome measures such as the EQ-5D (which can be used to estimate QALYs) are not routinely collected, a reliance on routine data alone would make it difficult to compare the benefits of the provision of training in supported communication skills to the benefits of other interventions on the same scale. We found that it was unnecessary to re-format the EQ-5D-3L as reported by Palmer et $a l,{ }^{46}$ for use with people with aphasia.

\section{CONCLUSIONS}

The study has highlighted issues that require consideration and modification before feasibility can be confirmed and a subsequent trial undertaken: (1) Cluster-randomisation of stroke units; (2) The use of routinely-collected patient clinical data (eg, TOMS) or proxy reports for SAQOL39g and EQ-5D-3L; (3) Conversation Partner Trainer involvement was perceived as extremely valuable and a key aspect of training; however, modifications to training should be made to accommodate different staff roles/levels of experience and work with people with severe aphasia and/or cognitive impairments; (4) a reliable approach to staff supported communication skills assessment must be devised.

Acknowledgements The authors acknowledge the contributions to the study design and conduct of others in the SCIP-R team: Helen Watson, Patricia White, John Mallett, Kneale Metcalf, Debbie Stanton and Iona Macrae. The authors acknowledge the interest and generous support of Aura Kagan and the Aphasia Institute, Toronto in enabling our use of the CAMS3 measure and resources for the Pocket Guide.

Collaborators On behalf of the study team: Supported Communication to Improve Participation in Rehabilitation of people with moderate-severe aphasia after stroke: a pilot study (SCIP-R).

Contributors $\mathrm{SH}$ was the $\mathrm{Cl}$ and led the design of the trial and drafted the article. AC, GB and VMP made substantial contributions to the concept, design and management of the study. $\mathrm{KL}$ was one of the RAs on the study and responsible for recruitment and data collection. All authors have read and approved the final manuscript.

Funding This trial was funded by the 06 (Grant Reference Number PB-PG-0609-17264). This article represents independent research; the views expressed are those of the authors and not necessarily those of the NHS, the NIHR or the Department of Health.

Competing interests None declared.

Ethics approval East of England NRES Committee, UK.

Provenance and peer review Not commissioned; externally peer reviewed.

Data sharing statement No additional data are available.

Open Access This is an Open Access article distributed in accordance with the Creative Commons Attribution Non Commercial (CC BY-NC 4.0) license, which permits others to distribute, remix, adapt, build upon this work noncommercially, and license their derivative works on different terms, provided the original work is properly cited and the use is non-commercial. See: http:// creativecommons.org/licenses/by-nc/4.0/

\section{REFERENCES}

1. Townsend N, Wickramasinghe K, Bhatnagar P, et al. Coronary heart disease statistics 2012 edition. London: British Heart Foundation, 2012.

2. Department of Health. National stroke strategy. London: Department of Health, 2007.

3. O'Halloran R, Worrall L, Hickson L. Environmental factors that influence communication between patients and their healthcare providers in acute hospital stroke units: an observational study. Int $J$ Lang Commun Disord 2011;46:30-47.

4. Simmons Mackie NN, Kagan A, O'Neill Christie C, et al. Communicative access and decision making for people with aphasia: implementing sustainable healthcare systems change. Aphasiology 2007;21:9-66.

5. Hemsley B, Werninck M, Worrall L. "That really shouldn't have happened": people with aphasia and their spouses narrate adverse events in hospital. Aphasiology 2013;27:706-22.

6. WHO. Patient safety workshop. Learning from error. Geneva: World Health Organisation, 2008.

7. NICE. Patient experience in adult NHS services: improving the experience of care for people using adult NHS services. Clinical guideline 138. London: National Clinical Guidelines Centre, 2012.

8. Jesus TS, Silva IL. Towards an evidence-based patient-provider communication in rehabilitation: linking communication elements to better rehabilitation outcomes. Clin Rehab 2016;30:315-28.

9. NICE. Stroke rehabilitation. Long term rehabilitation after stroke. Clinical guideline 162. London: National Clinical Guidelines Centre, 2013.

10. Kagan A, Black S, Duchan J, et al. Training volunteers as conversation partners using 'supported conversation for adults with aphasia (SCA)': a controlled trial. J Speech Lang Hear Res 2001;44:624-38.

11. Simmons-Mackie N, Raymer A, Armstrong E, et al. Communication partner training in aphasia: a systematic review. Arch Phys Med Rehabil 2010;91:1814-37.

12. Clark H, Wilkes-Gibbs D. Referring as a collaborative process. Cognition 1986;22:1-3.

13. Kagan A. Supported conversation for adults with aphasia: methods and resources for training conversation partners. Aphasiology 1998;12:816-30.

14. Craig P, Dieppe P, Macintyre S, et al. Developing and evaluating complex interventions: the new Medical Research Council guidance. BMJ 2008;337:a1655.

15. Jensen LR, Løvholt AP, Sørensen IR, et al. Implementation of supported conversation for communication between nursing staff and in-hospital patients with aphasia. Aphasiology 2015;29:57-80.

16. McGilton K, Sorin-Peters R, Sidani S, et al. Focus on communication: increasing the opportunity for successful staffpatient interactions. Int J Older People Nurs 2011;6:13-24.

17. NICE. How to change practice. Understand, identify and overcome barriers to change. London: National Institute for Health \& Clinical Excellence, 2007.

18. Heaven $\mathrm{C}$, Clegg J, Maguire P. Transfer of communication skills training from workshop to workplace: the impact of clinical supervision. Patient Educ Couns 2006;60:313-25.

19. Kagan A, Simmons-Mackie N, Rowland A, et al. Counting what counts: a framework for capturing real-life outcomes of aphasia intervention. Aphasiology 2007;22:258-80.

20. Shanyinde M, Pickering RM, Weatherall M. Questions asked and answered in pilot and feasibility randomized controlled trials. BMC Med Res Method 2011;11:117.

21. Medical Research Council. Cluster randomised controlled trials methodological and ethical considerations. MRC Clinical Trials Series, 2002.

22. Taljaard M, Weijer C, Grimshaw JM, et al. Ethical and policy issues in cluster randomized trials: rationale and design of a mixed methods research study. Trials 2009;10:61.

23. Enderby P, John A, Petheram B. Therapy outcome measures for rehabilitation professionals. 2nd edn. Chichester: John Wiley \& Sons, 2006.

24. Lancaster GA, Dodd S, Williamson PR. Design and analysis of pilot studies: recommendations for good practice. J Eval Clin Practice 2004; 10:307-12. 
25. Kagan A, Winkel J, Black S, et al. A set of observational measures for rating support and participation in conversation between adults with aphasia and their conversation partners. Top Stroke Rehab 2004;11:67-83.

26. Intercollegiate Stroke Working Party. National clinical guideline for stroke. 4th edn. London: Royal College of Physicians; 2012.

27. McVicker S, Parr S, Pound C, et al. The Communication Partner Scheme: a project to develop long-term, low-cost access to conversation for people living with aphasia. Aphasiology 2009;23:52-71.

28. Hilari K, Lamping DL, Smith SC, et al. Psychometric properties of the Stroke and Aphasia Quality of Life Scale (SAQOL-39) in a generic stroke population. Clin Rehab 2009;23:544-57.

29. Brooks R. EuroQol: the current state of play. Health Policy 1996;37:53-72.

30. Braun V, Clarke V. Using thematic analysis in psychology. Qual Res Psych 2006;3:77-101.

31. Sarangi S. Healthcare interaction as an expert communication system: an activity analysis perspective. In: Streeck J, ed. New adventures in language and interaction. Amsterdam: John Benjamins, 2010:167-97.

32. Heath C, Hindmarsh J. Analysing interaction: video, ethnography and situated conduct. In: May T, ed. Qualitative research in action. London: Sage, 2002:99-122.

33. Horton S, Lane K, Shiggins C. Supporting communication for people with aphasia in stroke rehabilitation: transfer of training in a multidisciplinary stroke team. Aphasiology 2016;30:629-56.

34. NICE. Guide to the methods of technology appraisal 2013. National Institute of Health and Clinical Excellence (NICE) publications, 2013.

35. McMullen $\mathrm{H}$, Griffiths $\mathrm{C}$, Leber W, et al. Explaining high and low performers in complex intervention trials: a new model based on diffusion of innovations theory. Trials 2015;16:242.
36. Hadidi N, Buckwalter K, Lindquist R, et al. Lessons learned in recruitment and retention of stroke survivors. J Neurosci Nurs 2012; 44:105-10.

37. Langhorne $P$, Stott DJ, Knight $A$, et al. Very early rehabilitation or intensive telemetry after stroke: a pilot randomised trial. Cerebrovasc Dis 2010;29:352-60.

38. Ali M, Bath PM, Lyden PD, et al. Representation of people with aphasia in randomized controlled trials of acute stroke interventions. Int J Stroke 2014;9:174-82.

39. Godecke E, Hird K, Lalor EE, et al. Very early post stroke aphasia therapy: a pilot randomized controlled efficacy trial. Int J Stroke 2012;7:635-44.

40. McKenna S, Jones F, Glenfield P, et al. Bridges self-management program for people with stroke in the community: a feasibility randomized controlled trial. Int J Stroke 2015;10:697-704.

41. Campbell GB, Skidmore ER, Whyte EM, et al. Overcoming practical challenges to conducting clinical research in the inpatient stroke rehabilitation setting. Top in Stroke Rehab 2015;22:386-95.

42. John A, Hughes A, Enderby P. Establishing clinician reliability using the therapy outcome measure for the purpose of benchmarking services. Adv in Sp Lang Path 2002;4:79-87.

43. Legg C, Young L, Bryer A. Training sixth-year medical students in obtaining case-history information from adults with aphasia. Aphasiology 2005;19:559-75.

44. Togher L, Power E, Tate R, et al. Measuring the social interactions of people with traumatic brain injury and their communication partners: the adapted Kagan scales. Aphasiology 2010;24:914-27.

45. Eriksson K, Bergström S, Carlsson E, et al. Aspects of rating communicative interaction: effects on reliability and agreement. $J$ Int Res Commun Disord 2014;5:245-67.

46. Palmer R, Enderby $\mathrm{P}$, Cooper $\mathrm{C}$, et al. Computer therapy compared with usual care for people with long-standing aphasia poststroke: a pilot randomized controlled trial. Stroke 2012;43:1904-11. 